\title{
Efecto de la proporción de mezclas biodiesel/petrodiesel en el desempeño mecánico-ambiental de motores
}

\author{
Alejandro A. Flórez-Montoya", Andrés F. Rojas ${ }^{*}$ \\ *Departamento de Ingeniería, Universidad Nacional de Colombia, Palmira \\ §email:afrojasgo@unal.edu.co
}

Resumen

(Recibido: Agosto 03 de 2009 - Aceptado: Diciembre 12 de 2009)

\begin{abstract}
En este articulo se reportan los resultados del desempeño mecánico y ambiental de un motor diesel estacionario (potencia nominal: $19 \mathrm{HP}$ ) y un motor diesel de un tractor (potencia nominal: $50.5 \mathrm{HP}$ ). Se utilizó combustible de diferentes mezclas de biodiesel provenientes de higuerilla, palma y de aceite de fritura usado (AFU), mezcladas con petrodiesel, en proporciones porcentuales de biodiesel/petrodiesel de 0/100 (D100), 10/90 (B10), 20/80 (B20) y 30/70 (B30). El desempeño mecánico se evaluó con un dinamómetro hidráulico, y las emisiones gaseosas se determinaron con un analizador de gases Orsat. Se evaluó la potencia mecánica, consumo específico de combustible, eficiencia, y porcentaje de $\mathrm{CO}, \mathrm{O}_{2}, \mathrm{CO}_{2}$, entre otros gases, para cada mezcla. Para el motor estacionario se encontró que la mezcla 10\% biodiesel de palma - 10\% biodiesel de higuerilla y 80\% de petrodiesel (PB10/HB10/D80) presentó reducción en el consumo de combustible, y composición de gases de combustión similar a D100, pero menor a la de $20 \%$ de diesel de palma - 80\% de petrodiesel (PB20/D80). En el motor diesel del tractor se encontró que la mezcla 5\% de diesel de palma - 5\% de diesel de higuerilla - 10\% de AFU y 80\% de petrodiesel (PB5/HB5/AFUB10/D80) presentó el mejor comportamiento en cuanto a potencia y consumo de combustible, con $32.35 \mathrm{~kW}$ y $224.83 \mathrm{~g} / \mathrm{kWh}$ respectivamente; mientras que la mezcla $3.33 \%$ de biodiesel de palma $-3.33 \%$ de biodiesel de higuerilla $-3.33 \%$ de biodiesel de AFU y 90\% de petrodiesel (PB3.33/HB3.33/AFUB3.33/D90) presentó mejor combustión comparada con 10\% de diesel de palma y 90\% de petrodiesel (PB10/D90), mostrando un aumento en la producción de CO2 y disminución en CO.
\end{abstract}

Palabras Claves: Biodiesel, Mezclas, Desempeño mecánico y ambiental, Motor ciclo diesel.

CHEMICAL ENGINEERING

\section{Effect of biodiesel/petrodiesel blends on the mechanical/environmental performance of engines}

\begin{abstract}
In this paper, the results of the mechanical and environmental performance of a stationary petrodiesel engine (rated power: $19 \mathrm{HP}$ ) and a diesel engine of a tractor (rated power: $50.5 \mathrm{HP}$ )are reported. Different mixtures of biodiesel from castor oil, palm oil and used frying oil (AFU), mixed with petrodiesel in percentage proportions of biodiesel/petrodiesel 0/100 (D100), 10/90 (B10), 20/80 (B20) and 30/70 (B30) were used. The mechanical performance was evaluated using a hydraulic dynamometer, and gaseous emissions were measured with an Orsat gas analyzer. The mechanical power, specific fuel consumption, efficiency, and percentage of $\mathrm{CO}, \mathrm{O}_{2}, \mathrm{CO}_{2}$ and other gases, for each mixture were determined. It was found that for the stationary engine, the mixture $10 \%$ palm diesel $10 \%$ castor diesel and $80 \%$ petrodiesel (PB10/HB10/D80) reported a reduction in fuel consumption, and the combustion gas composition is similar to D100, but lower than the mixture $20 \%$ palm diesel and $80 \%$ petrodiesel (PB20/D80) mixture. For the tractor diesel engine, it was found that the 5\% palm diesel - 5\% castor diesel - 10\% AFU and $80 \%$ petrodiesel (PB5/HB5/AFUB10/D80) mixture presented better performance in power and fuel consumption, with $32.35 \mathrm{~kW}$ and $224.83 \mathrm{~g} / \mathrm{kWh}$, respectively; while the $3.33 \%$ palm diesel $-3.33 \%$ castor diesel $3.33 \% \mathrm{AFU}$ and $90 \%$ petrodiesel (PB3.33/HB3.33/AFUB3.33/D90) mixture reported a better combustion compared to $10 \%$ palm diesel $-90 \%$ petrodiesel (PB10/D90), showing an increase in the production of $\mathrm{CO}_{2}$ and a decrease in CO production.
\end{abstract}

Keywords: Biodiesel, Blends, Mechanical environmental performance, Cycle diesel engine. 


\section{Introducción}

Ante la escalada del precio del petróleo, la vulnerabilidad al evidente agotamiento de los combustibles fósiles en un lapso relativamente corto, y la preocupación por el cuidado de la naturaleza y el ambiente, se ha generado gran interés en la búsqueda de nuevos carburantes que puedan reducir la dependencia existente de los derivados del petróleo, que sean más ecológicos y que generen menor cantidad de residuos. Durante los últimos años las investigaciones se han enfocado al estudio de los biocombustibles, que son catalogados como fuentes de energía renovables al proceder de diferentes formas de biomasa.

Una alternativa en cuanto a combustibles para motores de encendido por compresión (motores diesel) que ha producido impactos positivos en los países europeos y en los Estados Unidos, es el biodiesel, combustible proveniente de plantas oleaginosas, grasas animales, aceite de fritura usado e incluso de bacterias y microalgas (Kalscheuer et al., 2006; Issariyakul et al. 2008), y se caracteriza por ser biodegradable y no tóxico (Tinaut et al. 1999). Según Zhang et al. (2003) y Meher et al. (2006), el biodiesel se define como una mezcla de mono-alquíl ésteres de triglicéridos y de ácidos grasos de cadena larga derivados de aceites vegetales y/o grasas animales, que se obtiene principalmente por la transesterificación de éstos con un alcohol de cadena corta (metanol, etanol o propanol), en presencia de un catalizador ácido, básico o enzimático.

Un buen biodiesel se caracteriza por lograr que los motores no sufran mayores modificaciones ni presenten problemas a largo plazo; además de que la potencia y el consumo no se vean muy afectados. Muestra de ello es la utilización de biodiesel como combustible en vehículos convencionales a partir de 1998, salvo en modelos anteriores a este año, en los que se reemplaza los manguitos de goma (NBR) por un material más resistente (el biodiesel disuelve al NBR) (Herrero \& Maiza, 2008). Adicionalmente, las modificaciones al motor pueden darse por la utilización de un motor con pre-cámara, que son menos exigentes en cuanto a regulaciones, la adaptación del motor para precalentar el combustible a $60^{\circ} \mathrm{C}$ antes de la bomba de inyección, y la creación de un motor específico para quemar biodiesel (motores Elsbett) (Homer, 2006). Por otro lado, el deterioro del motor y su rendimiento, son muy similares al utilizar petrodiesel o mezclas de biodiesel/petrodiesel, y varían respecto a la materia prima utilizada para la producción del biodiesel (Agudelo et al., 2003) y al aumento en la proporción de biodiesel en la mezcla. Esto se debe al menor poder calorífico que tiene el biodiesel comparado con el petrodiesel. Además del poder calorífico, propiedades como la densidad y la viscosidad, tienen influencia sobre el rendimiento del motor y las emisiones gaseosas (Canakci et al., 2009).

Actualmente, las investigaciones realizadas sobre mezclas de biodiesel de diferentes fuentes que cumplan con los parámetros legales establecidos globalmente son escasas, una de ella es la mezcla de biodiesel de palma y jatrofa (Sarin et al., 2006). No obstante, también se han realizado algunas investigaciones en las que se obtiene biodiesel al mezclar aceites de diferentes fuentes vegetales, como es el caso de mezclas de aceite de fritura usado con aceite canola (Issariyakul et al., 2008), y mezclas de aceites de algodón, soya e higuerilla (Plentz et al., 2007).

En Colombia, la normatividad en biocombustibles se enfoca principalmente en el biodiesel de aceite de palma, dejando sin mercado al biodiesel proveniente de otras fuentes vegetales como el aceite de higuerilla, jatrofa e incluso aceite de fritura usado (AFU), generando monopolios alrededor de los cultivos de palma que no permite incentivar y aprovechar otros cultivos que ayuden al desarrollo energético del país y puedan servir como alternativa para la erradicación de cultivos ilícitos, y la utilización de residuos grasos de restaurantes, cafeterías y de empresas productoras de frituras, entre otros.

El objetivo de este artículo es mostrar el efecto de las mezclas de tres diferentes biodiesel con petrodiesel sobre el desempeño mecánico (potencia, eficiencia y consumo específico de combustible), y ambiental (emisiones gaseosas), 
de dos motores diesel (uno estacionario y uno de tractor). Además, se establece la mezcla biodiesel/petrodiesel, que cumpla con la reglamentación colombiana vigente (NTC 1438 y 5444), proporcione mejor desempeño mecánico (mayor potencia y menor consumo de combustible) y ambiental, permitiendo la entrada al mercado energético de otras fuentes alternas de generación de energía (higuerilla y aceite de fritura usado), y promover los policultivos energéticos, la sustitución de cultivos ilícitos y la reutilización de residuos grasos.

\section{Metodología}

\subsection{Materiales}

Para las mezclas se utilizó biodiesel de aceite de palma, higuerilla y aceite de fritura usado. El biodiesel de palma y de aceite de fritura usado se produjo con metanol, mientras que el aceite de higuerilla se obtuvo por etanólisis. Los tres biodiesel se prepararon por catálisis básica con $\mathrm{NaOH}$. El biodiesel de higuerilla fue preparado en el Laboratorio Biotecnología y Agroindustria de la Universidad Nacional de Colombia Sede Manizales; el biodiesel de aceite de fritura usado se preparó en el laboratorio de Fitoquímica de la Universidad Nacional de Colombia Sede Palmira; el biodiesel de palma fue donado por Cenipalma; y el petrodiesel se obtuvo en una estación de gasolina de la ciudad de Palmira. Se prepararon mezclas de biocombustible en proporciones de cero biodiesel (B0 o D100), 10\% (B10), 20\% (B20), y 30\% de mezclas de biodiesel (B30); en las que los porcentajes de 10,20 y $30 \%$, representan ya sea uno de los tres biodiesel (palma, higuerilla o AFU) o una mezcla entre ellos ya sea binaria o terciaria. En la Tabla 1 se presenta los porcentajes de mezcla de los biodiesel usados con su respectiva nomenclatura.

\subsection{Caracterización de las mezclas de Biodiesel/Petrodiesel}

Las mezclas combustibles preparadas se caracterizaron respecto a la viscosidad a $40^{\circ} \mathrm{C}$ (ASTM D445), índice de acidez (ASTM D664), punto de inflamación (ASTM D93), cenizas sulfatadas (ASTM D874) e índice de yodo
(HANUS), poder calorífico inferior (ASTM D240) y densidad a $15^{\circ} \mathrm{C}$ (ASTM D4052), ya que estos parámetros influyen en el desempeño del motor y el deterioro del mismo a largo plazo. De tal manera que una alta viscosidad y/o densidad conlleva a la colmatación de filtros y a una obstrucción de los inyectores, una acidez elevada podría conducir a problemas de corrosión, el punto de inflamación alto es ideal por cuestiones de seguridad y un poder calorífico bajo incrementa el consumo de combustible.

\subsection{Desempeño mecánico}

Para los ensayos del desempeño mecánico se utilizaron dos motores de ciclo diesel. El primero, un motor estacionario marca KIPOR con potencia nominal según el fabricante de $14 \mathrm{~kW}$ a 3,600 rpm y 2 cilindros en línea. El segundo, un motor de un tractor MASSEY-FERGUSON con potencia nominal de $37.5 \mathrm{~kW}$ a 2,000 rpm y 4 cilindros en línea. Los dos motores son de 4 tiempos y refrigerados por agua. Durante la fase experimental se evaluó el desempeño mecánico de cada motor, determinando sus parámetros característicos utilizando como combustible diferentes mezclas de biodiesel-petrodiesel (Tabla 1). Estas mezclas de biodiesel/petrodiesel se plantearon debido a que son proporciones que la legislación colombiana pretende implantar en los próximos 15 años. Las proporciones de las mezclas en todos los experimentos se realizaron en condiciones ambientales de la ciudad de Palmira, la cual se encuentra localizada a 1050 metros sobre el nivel del mar. En el motor estacionario se evaluó el consumo de combustible y la composición de las emisiones gaseosas sin aplicarle ningún tipo de carga al motor; mientras que en el tractor MASSEY-FERGUSON se estudió el torque, potencia, consumo específico de combustible, eficiencia y composición de las emisiones gaseosas, estos parámetros se midieron con una velocidad fija de 2100 RPM en promedio.

La potencia se evaluó mediante un dinamómetro hidráulico TAYLOR HI-EFF acoplado por medio de un cardán al eje trasero (toma de fuerza) del motor MASSEY-FERGUSON. Este dispositivo se basa en el principio de la disipación de energía en la fricción líquida. Para efectuar el cálculo de la velocidad y del par torsor del motor se analizó el 
Ingeniería y Competitividad, Volumen 11, No. 2, p. 63 - 78 (2009)

Tabla 1. Relación de mezclas usadas en los experimentos

\begin{tabular}{|c|c|}
\hline Denominación & Composición \\
\hline $\mathrm{B} 0=\mathrm{D} 100$ & $100 \%$ Petrodiesel convencional (muestra blanco) \\
\hline PB10-D90 & $10 \%$ Biodiesel de Palma y $90 \%$ Petrodiesel \\
\hline HB10-D90 & $10 \%$ Biodiesel de Higuerilla y $90 \%$ Petrodiesel \\
\hline AFUB10-D90 & $10 \%$ Biodiesel de AFU y $90 \%$ Petrodiesel \\
\hline PB5-HB5-D90 & 5\% Biodiesel de Palma / 5\% Biodiesel de Higuerilla / 90\% Petrodiesel \\
\hline HB5-AFUB5-D90 & 5\% Biodiesel de Higuerilla / 5\% Biodiesel deAFU / 90\% Petrodiesel \\
\hline PB5-AFUB5-D90 & 5\% Biodiesel de Palma / 5\% Biodiesel de AFU / 90\% Petrodiesel \\
\hline PB5-HB2.5-AFUB2.5-D90 & 5\% Biodiesel de Palma / 2.5\% Biodiesel de Higuerilla / 2.5\% Biodiesel de AFU / 90\% Petrodiesel \\
\hline PB2.5-HB5-AFUB2.5-D90 & 2.5\% Biodiesel de Palma / 5\% Biodiesel de Higuerilla / 2.5\% Biodiesel de AFU / 90\% Petrodiesel \\
\hline PB2.5-HB2.5-AFUB5-D90 & 2.5\% Biodiesel de Palma / 2.5\% Biodiesel de Higuerilla / 5\% Biodiesel de AFU / 90\% Petrodiesel \\
\hline PB3.33-HB3.33-AFUB3.33-D90 & $\begin{array}{c}\text { 3.33\% Biodiesel de Palma / 3.33\% Biodiesel de Higuerilla / 3.33\% Biodiesel de AFU / 90\% } \\
\text { Petrodiesel }\end{array}$ \\
\hline PB20-D80 & $20 \%$ Biodiesel de Palma y $80 \%$ Petrodiesel \\
\hline HB20-D80 & $20 \%$ Biodiesel de Higuerilla y $80 \%$ Petrodiesel \\
\hline AFUB20-D80 & $20 \%$ Biodiesel de AFU y $80 \%$ Petrodiesel \\
\hline PB10-HB10-D80 & $10 \%$ Biodiesel de Palma / 10\% Biodiesel de Higuerilla / $80 \%$ Petrodiesel \\
\hline PB10-AFUB10-D80 & 10\% Biodiesel de Palma / 10\% Biodiesel de AFU / 80\% Petrodiesel \\
\hline HB10-AFUB10-D80 & 10\% Biodiesel de Higuerilla / 10\% Biodiesel de AFU / $80 \%$ Petrodiesel \\
\hline PB10-HB5-AFUB5-D80 & 10\% Biodiesel de Palma / 5\% Biodiesel de Higuerilla / 5\% Biodiesel de AFU / 80\% Petrodiesel \\
\hline PB5-HB10-AFUB5-D80 & 5\% Biodiesel de Palma / 10\% Biodiesel de Higuerilla / 5\% Biodiesel de AFU / 80\% Petrodiesel \\
\hline PB5-HB5-AFUB10-D80 & 5\% Biodiesel de Palma / 5\% Biodiesel de Higuerilla / 10\% Biodiesel de AFU / 80\% Petrodiesel \\
\hline PB6.66-HB6.66-AFUB6.66-D80 & $\begin{array}{c}\text { 6.66\% Biodiesel de Palma / 6.66\% Biodiesel deHiguerilla / 6.66\% Biodiesel de AFU / 80\% } \\
\text { Petrodiesel }\end{array}$ \\
\hline PB30-D70 & $30 \%$ Biodiesel de Palma y $70 \%$ Petrodiesel \\
\hline HB30-D70 & $30 \%$ Biodiesel de Higuerilla y $70 \%$ Petrodiesel \\
\hline AFUB30-D70 & $30 \%$ Biodiesel de AFU y $70 \%$ Petrodiesel \\
\hline PB15-HB15-D70 & 15\% Biodiesel de Palma / 15\% Biodiesel Higuerilla / 70\% Petrodiesel \\
\hline PB15-AFUB15-D70 & 15\% Biodiesel de Palma / 15\% Biodiesel. AFU / 70\% Petrodiesel \\
\hline HB15-AFUB15-D70 & 15\% Biodiesel Higuerilla / 15\% Biodiesel. AFU / 70\% Petrodiesel \\
\hline PB15-HB7.5-AFUB7.5-D70 & 15\% Biodiesel de Palma / 7.5\% Biodiesel de Higuerilla / 7.5\% Biodiesel de AFU / 70\% Petrodiesel \\
\hline PB7.5-HB15-AFUB7.5-D70 & 7.5\% Biodiesel de Palma / 15\% Biodiesel de Higuerilla / 7.5\% Biodiesel de AFU / 70\% Petrodiesel \\
\hline PB7.5-HB7.5-AFUB15-D70 & 7.5\% Biodiesel de Palma / 7.5\% Biodiesel de Higuerilla / 15\% Biodiesel de AFU / 70\% Petrodiesel \\
\hline PB10-HB10-AFUB10-D70 & $10 \%$ Biodiesel de Palma / 10\% Biodiesel de Higuerilh / 10\% Biodiesel de AFU / 70\% Petrodiesel \\
\hline
\end{tabular}




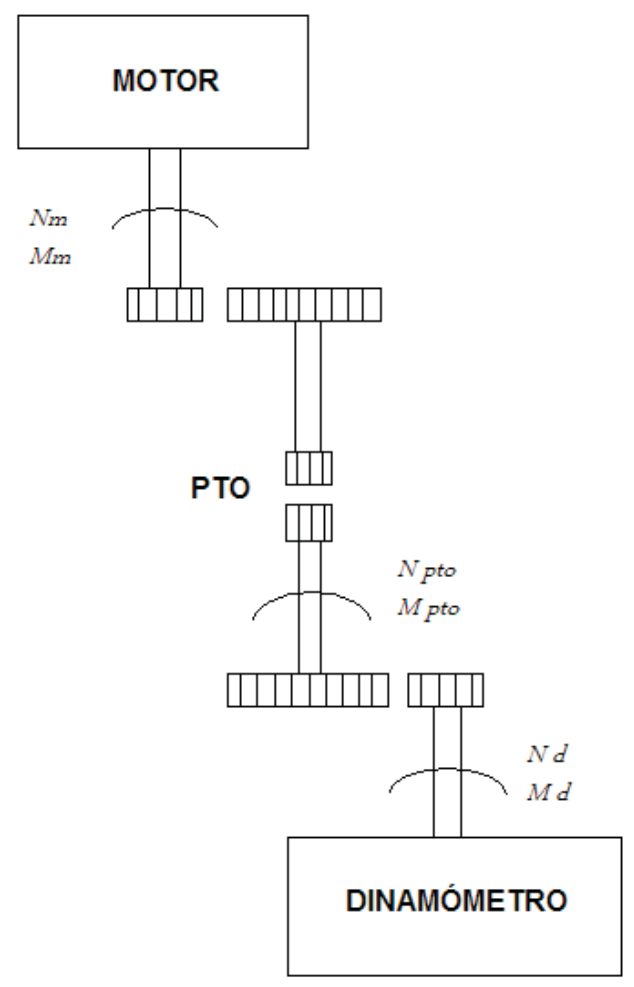

Figura 1. Esquema del sistema de transmisión

sistema de trasmisión entre el motor y el dinamómetro durante las pruebas de funcionamiento (Figura 1). A partir de la figura 1 se determinaron las siguientes relaciones:

$$
\begin{aligned}
& i=\frac{N_{p t o}}{N_{d}}=\frac{M_{d}}{M_{p t o}}=0.2926 \\
& i_{m}=\frac{N_{m}}{N_{p t o}}=\frac{M_{p t o}}{M_{m}}=3.89
\end{aligned}
$$

Donde $i$ es la relación de engranajes entre el dinamómetro y la toma de fuerza, $i_{m}$ es la relación de engranajes entre la toma de fuerza y el motor del tractor, Npto es la velocidad en la toma de fuerza (rpm), $N d$ es la velocidad del dinamómetro (rpm), $M_{d}$ es el par torsor del dinamómetro (Nm), Mpto es el par torsor de la toma de muestra $(\mathrm{Nm})$, $\mathrm{Nm}$ es la velocidad del motor (rpm), y $\mathrm{Mm}$ es el par torsor del motor $(\mathrm{Nm})$.
Para determinar la potencia del motor, primero se calcula el par torsor del dinamómetro el cual se halla multiplicando la fuerza en el dinamómetro por su brazo (eje central hasta la bomba hidráulica, $0.34 \mathrm{~m}$ ), conociendo éste y la velocidad registrada en el mismo, se calcula la velocidad y el par torsor en la toma de fuerza y posteriormente en el motor utilizando las Ec. (1) y (2), y luego se cálculo la potencia por medio de la Ec. (3).

$$
P=\frac{M_{m} N_{m}}{9550}
$$

Donde P es la potencia del motor $(\mathrm{kW})$. El consumo específico de combustible es un indicativo de la potencia entregada por cada gramo de combustible en condiciones determinadas, y se halla empleando la Ec. (4).

$$
C E C=\frac{(c c)\left(\rho_{\text {mezcla }}\right)}{\tau P}
$$


Donde $C E C$ es el consumo específico de combustible $(\mathrm{g} / \mathrm{kWh}), c c$ es el consumo de combustible $\left(\mathrm{cm}^{3}\right), \rho$ es la densidad de la mezcla a $15^{\circ} \mathrm{C}\left(\mathrm{g} / \mathrm{cm}^{3}\right)$, y $t$ es el tiempo de funcionamiento del motor para un $c c$ de $100 \mathrm{~cm}^{3}(\mathrm{~h})$.

La eficiencia es un factor inversamente proporcional al consumo específico de combustible, está expresada en porcentaje, y da una idea de que tan eficiente es la combustión de una mezcla de combustibles en un motor. Se calcula por medio de la Ec. (5).

$$
E=\frac{\text { conversión ideal }}{\text { conversión real }}=\frac{3600}{(C E C)(H)}
$$

Donde $E$ es la eficiencia del motor, y $H$ el poder calorífico inferior del combustible $(\mathrm{kJ} / \mathrm{kg})$. Todos los resultados de las potencias generadas por las mezclas se comparan con la generada utilizando
D100 y PB/D (PB10, PB20 y PB30) como combustible. Además, es importante tener en cuenta el modelo, afinación, edad y tipo de motor, entre otros aspectos, al momento de comparar los resultados encontrados en esta investigación con los reportados en la literatura, ya que los valores del desempeño mecánico de un motor dependen tanto de la calidad de las mezclas como de las características del motor.

\subsection{Análisis de las emisiones gaseosas}

Se determinó la composición de los gases generados en la combustión de las diferentes mezclas de los combustibles evaluados mediante un analizador de gases Orsat, el cual sirvió para establecer los porcentajes de $\mathrm{CO}_{2}, \mathrm{O}_{2}, \mathrm{CO}$ y un conjunto de otros gases (hidrocarburos no quemados, $\mathrm{NO}_{\mathrm{x}}, \mathrm{SO}_{\mathrm{x}}, \mathrm{N}_{2}$, entre otros), presentes en cada tratamiento. Este equipo se basa en un análisis volumétrico restringido a la medición de volúmenes de gases, donde se toma una muestra de gas de $100 \mathrm{ml}$, a presión y temperatura ambiente.

Tabla 2. Caracterización de las mezclas Biodiesel/ Petrodiesel

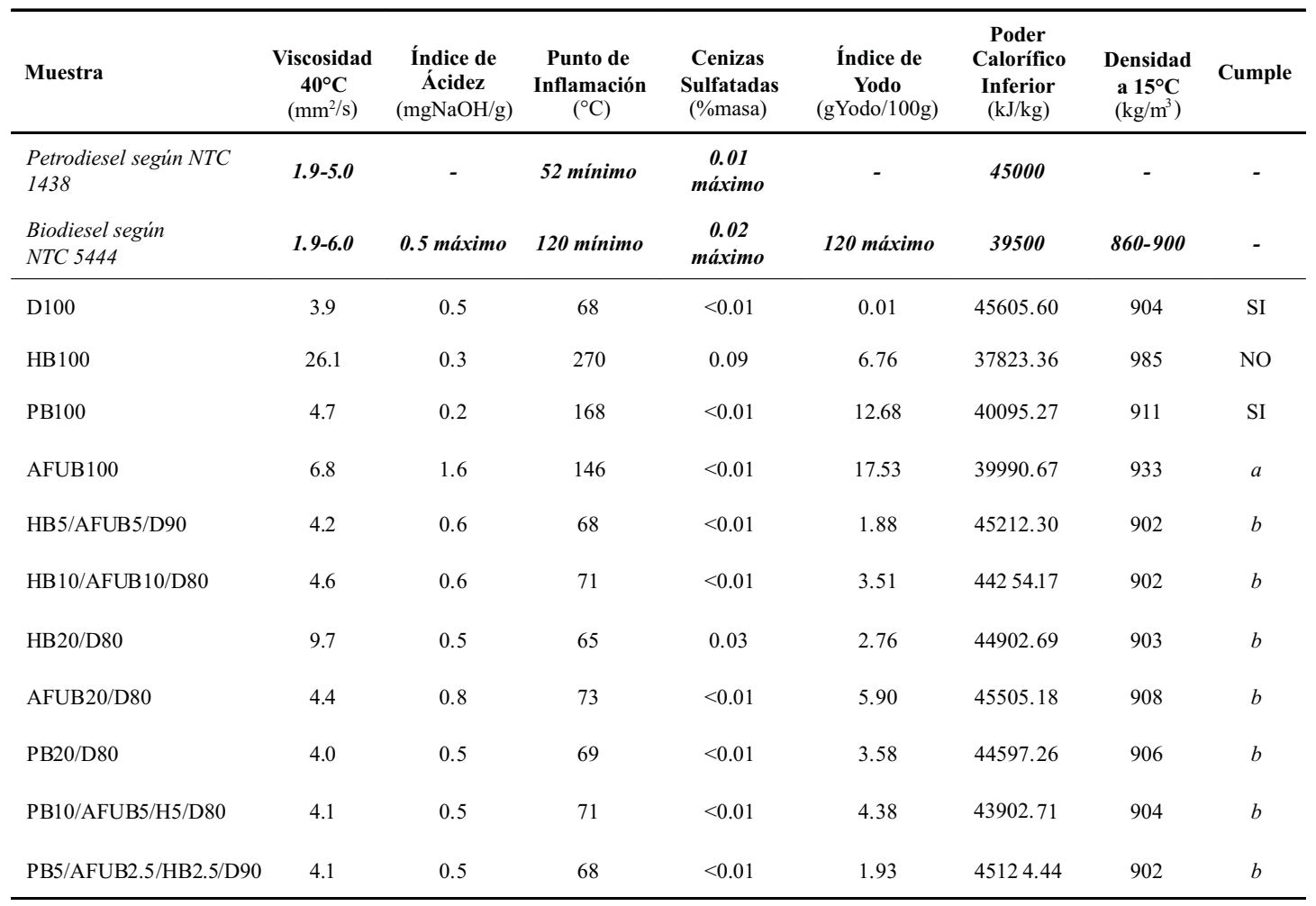

$a$ : Cumple con todos los parámetros a excepción del índice de Acidez.

$b$ : No cumplen con lo estipulado por la NTC 5444 debido a que esta norma aplica para el Biodiesel puro que va a ser mezclado con

Petrodiesel y en este caso los análisis se efectuaron a las mezclas ya preparadas, en consecuencia los datos reportados se asemejan más

a lo contemplado en la NTC 1438 (Petrodiesel puro) que a la NTC 5444. 


\section{Resultados y discusión}

\subsection{Caracterización de las mezclas de biodiesel/petrodiesel}

En la Tabla 2 se presentan los resultados de viscosidad, índice de acidez, punto de inflamación, cenizas sulfatadas, índice de yodo, poder calorífico inferior y densidad, de algunas mezclas de biocombustibles. Al comparar los resultados de la tabla para el petrodiesel (D100), biodiesel (B100) y sus mezclas, se encontró que el petrodiesel cumple con lo estipulado en la NTC 1438 al igual que el Biodiesel de Palma (PB100) cumple con los valores contemplados en la NTC 5444 que va a ser mezclado con petrodiesel, pero el B100 de Higuerilla (HB100) no cumple con los parámetros de viscosidad y cenizas sulfatadas con valores muy superiores a lo estipulado por la NTC 5444, lo cual puede conllevar a una colmatación de filtros y a obstrucciones en los inyectores del motor, además cuenta con un poder calorífico bajo comparado con los otros biodiesel, lo que representa un mayor consumo de este tipo de combustible por parte del motor. El B100 de AFU (AFUB100) también presentó problemas en el cumplimiento de los parámetros de viscosidad e índice de acidez con valores por encima de lo permitido, esto puede generar problemas de corrosión en el motor. Sin embargo, al evaluar las mezclas que contienen estos tipos de biodiesel se encontró que la mayoría de estas presentan una gran similitud con los valores de PB20/D80 que es la mezcla que regirá en Colombia a partir del $1^{0}$ de enero de 2012, según Decreto No. 2629 de julio 10 de 2007 del Ministerio de Minas y Energía. Por consiguiente, es clara la viabilidad de producir dichas mezclas, dada la gran similitud con PB20/D80 y el cumplimiento de la normatividad colombiana.

En relación a las mezclas de los diferentes biodiesel, se encontró que la mezcla de mayor viscosidad fue HB20/D80 con $9.7 \mathrm{~mm}^{2} / \mathrm{s}$, esto es apenas lógico ya que el BH100 cuenta con una viscosidad alta $\left(26.1 \mathrm{~mm}^{2} / \mathrm{s}\right)$; mientras que la mezcla de menor viscosidad fue PB20/D80 con 4.0 $\mathrm{mm}^{2} / \mathrm{s}$, este valor se encuentra dentro del rango permitido en la NTC 5444. En cuanto al índice de acidez la mezcla AFUB20/D80 tiene el mayor valor con $0.8 \mathrm{mg} \mathrm{NaOH} / \mathrm{g}$, las demás poseen valores aceptables de $0.5 \mathrm{mg} \mathrm{NaOH} / \mathrm{g}$ en promedio. El valor del punto de inflamación para la mezcla AFUB20/D80 es de $73^{\circ} \mathrm{C}$, mientras la mezcla HB20/D80 se inflama a $65^{\circ} \mathrm{C}$. En cuanto a cenizas sulfatadas todas las mezclas poseen valores menores a $0.01 \%$ (p/p) exceptuando la mezcla HB20/D80 con $0.03 \%(\mathrm{p} / \mathrm{p})$. El índice de yodo fue el parámetro de mayor variación entre las mezclas, presentándose el mayor valor para AFUB20/D80 con $5.9 \mathrm{~g}$ Yodo/100g y el menor valor para PB5/AFUB2.5/HB2.5/D90 con $1.93 \mathrm{~g}$ Yodo/100g. Los valores del poder calorífico son similares para todas las mezclas, alrededor de $44000 \mathrm{~kJ} / \mathrm{kg}$, que es un valor aceptable dentro de lo estipulado por la normatividad colombiana. Los valores de densidad a $15^{\circ} \mathrm{C}$ estuvieron levemente por encima de $900 \mathrm{~kg} / \mathrm{m}^{3}$, que es el máximo permitido en la NTC 5444. Es importante anotar que estas comparaciones se realizaron respecto a la norma NTC 5444, que se aplica a biodiesel puro, y a la norma NTC 1438, para petrodiesel, dado que no existe una norma para la mezcla biodiesel/petrodiesel.

\subsection{Desempeño mecánico motor estacionario KIPOR}

En el motor KIPOR se evaluó la composición de los gases de ignición y el consumo de combustible $(\mathrm{ml} / \mathrm{s})$ a 2213 RPM en promedio. Los resultados obtenidos se presentan en la Tabla 3. En cuanto a la composición de los gases, las mezclas en general muestran un incremento relativamente alto en la producción de $\mathrm{CO}$ comparando con la combustión del D100, esto indica que la combustión es incompleta en todos los casos, siendo mayor para la mezcla PB10/AFUB10/D80 con $7.08 \%$ de CO. Por el contrario, la mezcla que evidenció ser la menos incompleta en la combustión fue PB10/HB10/D80 con $2.11 \%$ de $\mathrm{CO}$, aunque este porcentaje sigue siendo mayor al D100, pero menor respecto a la mezcla comercial de PB20/D80. En cuanto a la producción de $\mathrm{CO}_{2}$ se detecta un leve incremento en la mayoría de las mezclas exceptuando la mezcla de AFUB20/D80 con $1.5 \%$ de $\mathrm{CO}_{2}$ comparada con el $2.25 \%$ del D100 y el $2.9 \%$ de PB20/D80. La mezcla PB10/HB10/D80 se destaca debido a que presenta la menor producción de $\mathrm{CO}$ con un leve incremento en el $\mathrm{CO}_{2}$ de $0.7 \%$ respecto al $\mathrm{D} 100$ y de $0.65 \%$ respecto 
Tabla 3. Resultados de las pruebas en el motor estacionario KIPOR

\begin{tabular}{lcccccc}
\hline Mezcla & $\mathbf{C O}_{2}(\%)$ & $\mathbf{O}_{2}(\mathbf{\%})$ & $\mathbf{C O}(\%)$ & $\begin{array}{c}\text { Otros } \\
\text { Gases }(\%)\end{array}$ & $\begin{array}{c}\text { Consumo } \\
(\mathbf{m l} / \mathbf{s})\end{array}$ & RPM \\
\hline PB20-D80 & 2.90 & 4.84 & 4.49 & 87.77 & 0.341 & 2266.50 \\
HB20-D80 & 2.65 & 4.88 & 5.08 & 87.40 & 0.318 & 2198.00 \\
AFUB20-D80 & 1.50 & 6.85 & 6.54 & 85.11 & 0.323 & 2199.50 \\
PB10-HB10-D80 & 2.95 & 2.52 & 2.11 & 92.41 & 0.306 & 2214.00 \\
PB10-AFUB10-D80 & 2.45 & 5.89 & 7.08 & 84.57 & 0.326 & 2220.00 \\
HB10-AFUB10-D80 & 2.50 & 3.59 & 3.51 & 90.40 & 0.319 & 2245.50 \\
PB10-HB5-AFUB5-D80 & 2.80 & 4.63 & 4.80 & 87.77 & 0.310 & 2207.50 \\
PB5-HB10-AFUB5-D80 & 2.95 & 4.07 & 3.54 & 89.44 & 0.359 & 2217.00 \\
PB5-HB5-AFUB10-D80 & 2.50 & 3.08 & 2.75 & 91.67 & 0.344 & 2186.50 \\
PB6.66-HB6.66-AFUB6.66-D80 & 2.90 & 4.84 & 4.38 & 87.88 & 0.332 & 2187.50 \\
D100 & 2.25 & 2.46 & 1.84 & 93.46 & 0.332 & 2204.00 \\
\hline
\end{tabular}

a PB20/D80. En todos los casos los resultados obtenidos muestran un comportamiento contrario a lo reportado por Barbosa et al. (2008), quienes observaron un decrecimiento importante de los porcentajes de $\mathrm{CO}$ y $\mathrm{CO}_{2}$ cuando aumenta la proporción de biodiesel en la mezcla, esto se puede explicar teniendo en cuenta el tipo de motor, la afinación del mismo y la calidad del aire de admisión.

Respecto al consumo de combustible, la mezcla $\mathrm{PB} 5 / \mathrm{HB} 10 / \mathrm{AFUB} 5 / \mathrm{D} 80$ reporta el mayor valor con un incremento del $8.13 \%$ respecto al D100 y de $5.20 \%$ respecto a PB20/D80, esto se debe básicamente al menor poder calorífico del biodiesel comparado con el petrodiesel. Mientras que la mezcla PB10/HB10/D80 muestra disminución en el consumo del $7.83 \%$ respecto al D100 y $10.26 \%$ respecto a PB20/D80, esto se debe a que la mezcla contiene HB10 aumentado su densidad y viscosidad, ya que este biodiesel presenta los valores más altos de estos dos parámetros en todas las muestras evaluadas. Esto causa problemas de fluidez, y a nivel de los inyectores genera problemas en su aspersión al interior de la cámara de combustión (Allen \& Watts, 2000). Al comparar los dos aspectos evaluados (composición de gases y consumo de combustible) la mezcla PB10/HB10/D80 presenta el menor consumo de combustible y una composición de gases de combustión similar a la del D100 y menor a la de PB20/D80.

\subsection{Motor agrícola MASSEY-FERGUSON}

A partir de los datos obtenidos durante el análisis del desempeño mecánico del motor y utilizando las ecuaciones descritas en la metodología, se determinó la potencia del motor, consumo específico de combustible y eficiencia, para cada mezcla de combustible. Estos resultados se presentan en las Tablas 4, 5 y 6 para las mezclas $\mathrm{B} 10, \mathrm{~B} 20$ y B30 respectivamente.

Potencia (P). En la evaluación de este parámetro se observa (Tablas 4, 5 y 6) que en términos generales disminuye, pero sin una tendencia clara a medida que se aumenta la proporción de 
Ingeniería y Competitividad, Volumen 11, No. 2, p. 63 - 78 (2009)

Tabla 4. Características de funcionamiento del motor alimentado con mezclas de B10

\begin{tabular}{|c|c|c|c|c|c|c|c|c|c|c|}
\hline Mezcla & $\begin{array}{l}\text { Nd } \\
(\mathbf{R P M})\end{array}$ & $\begin{array}{l}\text { Md } \\
(\mathbf{N m})\end{array}$ & $\begin{array}{l}\text { Npto } \\
\text { (RPM) }\end{array}$ & $\begin{array}{l}\text { Mpto } \\
\text { (Nm) }\end{array}$ & $\begin{array}{l}\text { Nm } \\
(\mathbf{R P M})\end{array}$ & $\begin{array}{l}\text { Torque } \\
\text { Mm } \\
\text { (Nm) }\end{array}$ & $\begin{array}{l}\text { Potencia } \\
\text { (kW) }\end{array}$ & $\underset{(\mathrm{g} / \mathrm{Kwh})}{\text { CEC }}$ & $\begin{array}{c}\text { Poder } \\
\text { Calorífico } \\
\text { Superior } \\
\text { (kJ/kg) }\end{array}$ & E (\%) \\
\hline D100 & 2100 & 145.19 & 614.46 & 496.20 & 2390.25 & 127.56 & 31.93 & 228.66 & 45605.60 & 34.52 \\
\hline PB10-D90 & 2100 & 117.97 & 614.46 & 403.17 & 2390.25 & 103.64 & 25.94 & 240.50 & 45054.56 & 33.22 \\
\hline HB10-D90 & 2150 & 124.02 & 629.09 & 423.84 & 2447.16 & 108.96 & 27.92 & 235.22 & 44827.37 & 34.14 \\
\hline AFUB10-D90 & 2200 & 87.72 & 643.72 & 299.79 & 2504.07 & 77.07 & 20.21 & 273.38 & 45044.10 & 29.23 \\
\hline PB5-HB5-D90 & 2150 & 95.28 & 629.09 & 325.63 & 2447.16 & 83.71 & 21.45 & 279.15 & 44940.97 & 28.70 \\
\hline HB5-AFUB5-D90 & 2150 & 117.97 & 629.09 & 403.17 & 2447.16 & 103.64 & 26.56 & 227.12 & 45212.30 & 35.06 \\
\hline PB5-AFUB5-D90 & 2100 & 140.65 & 614.46 & 480.70 & 2390.25 & 123.57 & 30.93 & 235.74 & 45049.33 & 33.90 \\
\hline $\begin{array}{l}\text { PB5-HB2.5-AFUB2.5- } \\
\text { D90 }\end{array}$ & 2100 & 151.24 & 614.46 & 516.88 & 2390.25 & 132.87 & 33.26 & 231.13 & 45124.44 & 34.52 \\
\hline $\begin{array}{l}\text { PB2 .5-HB5-AFUB2.5- } \\
\text { D90 }\end{array}$ & 2100 & 136.11 & 614.46 & 465.19 & 2390.25 & 119.59 & 29.93 & 218.86 & 44938.35 & 36.60 \\
\hline $\begin{array}{l}\text { PB2.5-HB2.5-AFUB5- } \\
\text { D90 }\end{array}$ & 1950 & 149.73 & 570.57 & 511.71 & 2219.52 & 131.55 & 30.57 & 240.36 & 44992.53 & 33.29 \\
\hline $\begin{array}{l}\text { PB3.33-HB3.33- } \\
\text { AFUB3.33-D90 }\end{array}$ & 2000 & 152.75 & 585.2 & 522.05 & 2276.43 & 134.20 & 31.99 & 236.79 & 41437.67 & 36.69 \\
\hline
\end{tabular}

Tabla 5. Características de funcionamiento del motor alimentado con mezclas de B20

\begin{tabular}{|c|c|c|c|c|c|c|c|c|c|c|}
\hline Mezcla & $\begin{array}{l}\text { Nd } \\
(\mathrm{RPM})\end{array}$ & $\begin{array}{l}\text { Md } \\
(\mathbf{N m})\end{array}$ & $\begin{array}{l}\text { Npto } \\
\text { (RPM) }\end{array}$ & $\begin{array}{l}\text { Mpto } \\
\text { (Nm) }\end{array}$ & $\begin{array}{l}\mathrm{Nm} \\
(\mathrm{RPM})\end{array}$ & $\begin{array}{c}\text { Torque } \\
\text { Mm } \\
\text { (Nm) }\end{array}$ & $\begin{array}{l}\text { Potencia } \\
(\mathrm{kW})\end{array}$ & $\begin{array}{c}\text { CEC } \\
(\mathrm{g} / \mathrm{kWh})\end{array}$ & $\begin{array}{c}\text { Poder } \\
\text { Calorífico } \\
\text { Superior } \\
(\mathbf{k J} / \mathbf{k g}) \\
\end{array}$ & E (\%) \\
\hline D100 & 2100 & 145.19 & 614.46 & 496.20 & 2390.25 & 127.56 & 31.93 & 228.66 & 45605.60 & 34.52 \\
\hline PB20-D80 & 2100 & 140.65 & 614.46 & 480.70 & 2390.25 & 123.57 & 30.93 & 213.80 & 44597.25 & 37.76 \\
\hline HB20-D80 & 2075 & 127.04 & 607.14 & 434.18 & 2361.79 & 111.61 & 27.60 & 279.27 & 44049. 15 & 29.26 \\
\hline AFUB20-D80 & 1900 & 155.78 & 555.94 & 532.39 & 2162.61 & 136.86 & 30.99 & 248.11 & 45505.18 & 31.89 \\
\hline PB10-HB10-D80 & 2150 & 101.33 & 629.09 & 346.31 & 2447.16 & 89.03 & 22.81 & 244.03 & 44276.34 & 33.32 \\
\hline PB10-AFUB10-D80 & 2075 & 137.63 & 607.14 & 470.36 & 2361.79 & 120.92 & 29.90 & 259.13 & 44493.07 & 31.22 \\
\hline HB10-AFUB10-D80 & 2100 & 101.33 & 614.46 & 346.31 & 2390.25 & 89.03 & 22.28 & 265.13 & 44254.16 & 30.68 \\
\hline $\begin{array}{l}\text { PB10-HB5-AFUB5- } \\
\text { D80 }\end{array}$ & 2150 & 111.92 & 629.09 & 382.49 & 2447.16 & 98.33 & 25.20 & 278.67 & 43902.71 & 29.43 \\
\hline $\begin{array}{l}\text { PB5-HB10-AFUB5- } \\
\text { D80 }\end{array}$ & 2150 & 98.31 & 629.09 & 335.97 & 2447.16 & 86.37 & 22.13 & 248.32 & 44271.11 & 32.75 \\
\hline $\begin{array}{l}\text { PB5-HB5-AFUB10- } \\
\text { D80 }\end{array}$ & 2150 & 143.68 & 629.09 & 491.04 & 2447.16 & 126.23 & 32.35 & 224.83 & 44379.47 & 36.08 \\
\hline $\begin{array}{l}\text { PB6.66-HB6.66- } \\
\text { AFUB6.66-D80 }\end{array}$ & 2175 & 93.77 & 636.40 & 320.47 & 2475.62 & 82.38 & 21.36 & 260.54 & 37269.75 & 37.07 \\
\hline
\end{tabular}


Ingeniería y Competitividad, Volumen 11, No. 2, p. 63 - 78 (2009)

Tabla 6. Características de funcionamiento del motor alimentado con mezclas de B30

\begin{tabular}{|c|c|c|c|c|c|c|c|c|c|c|}
\hline Mezcla & $\begin{array}{l}\text { Nd } \\
\text { (RPM) }\end{array}$ & $\begin{array}{l}\text { Md } \\
(\mathbf{N m})\end{array}$ & $\begin{array}{l}\text { Npto } \\
\text { (RPM) }\end{array}$ & $\begin{array}{l}\text { Mpto } \\
\text { (Nm) }\end{array}$ & $\begin{array}{l}\mathrm{Nm} \\
\text { (RPM) }\end{array}$ & $\begin{array}{c}\text { Torque } \\
\text { Mm } \\
\text { (Nm) }\end{array}$ & $\begin{array}{c}\text { Potencia } \\
\text { (Kw) }\end{array}$ & $\begin{array}{c}\text { CEC } \\
(\mathrm{g} / \mathrm{Kwh})\end{array}$ & $\begin{array}{c}\text { Poder } \\
\text { Calorífico } \\
\text { Superior } \\
(\mathbf{k J} / \mathbf{k g}) \\
\end{array}$ & E (\%) \\
\hline D100 & 2100 & 145.19 & 614.46 & 496.20 & 2390.25 & 127.56 & 31.93 & 228.66 & 45605.60 & 34.52 \\
\hline PB30-D70 & 2100 & 136.11 & 614.46 & 465.19 & 2390.25 & 119.59 & 29.93 & 249.21 & 43952.50 & 32.87 \\
\hline HB30-D70 & 1875 & 145.19 & 548.62 & 496.20 & 2134. 15 & 127.56 & 28.51 & 242.34 & 43270.92 & 34.33 \\
\hline AFUB30-D70 & 2025 & 148.97 & 592.51 & 509.13 & 2304.88 & 130.88 & 31.59 & 244.67 & 43921.12 & 33.50 \\
\hline PB15-HB15-D70 & 2000 & 145.19 & 585.20 & 496.20 & 2276.43 & 127.56 & 30.41 & 235.83 & 43611.71 & 35.00 \\
\hline PB15-AFUB15-D70 & 2075 & 128.55 & 607.14 & 439.35 & 2361.79 & 112.94 & 27.93 & 259.04 & 43936. 81 & 31.63 \\
\hline HB15-AFUB15-D70 & 1850 & 152.75 & 541.31 & 522.05 & 2105.70 & 134.20 & 29.59 & 224.54 & 43596.02 & 36.78 \\
\hline $\begin{array}{l}\text { PB15-HB7.5-AFUB7.5- } \\
\text { D70 }\end{array}$ & 2000 & 146.70 & 585.20 & 501.37 & 2276.43 & 128.89 & 30.72 & 224.32 & 43774. 26 & 36.66 \\
\hline $\begin{array}{l}\text { PB7.5-HB15-AFUB7.5- } \\
\text { D70 }\end{array}$ & 2025 & 145.95 & 592.51 & 498.79 & 2304.88 & 128.22 & 30.95 & 227.47 & 43603.86 & 36.30 \\
\hline $\begin{array}{l}\text { PB7.5-HB7.5-AFUB15- } \\
\text { D70 }\end{array}$ & 1975 & 147.46 & 577.88 & 503.96 & 2247.97 & 129.55 & 30.50 & 237.82 & 43766.41 & 34.59 \\
\hline $\begin{array}{l}\text { PB10-HB10-AFUB10- } \\
\text { D70 }\end{array}$ & 1950 & 148.21 & 570.57 & 506.54 & 2219.52 & 130.22 & 30.26 & 236.40 & 43714.85 & 34.84 \\
\hline
\end{tabular}

biodiesel en las mezclas (B10, B20, B30), como se ha observado en otros trabajos (Barbosa et. al., 2008; Hilbert et. al., 2006; Correa et. al., 2008). Por el contrario las mezclas B30 presentaron menor reducción de la potencia en términos generales, lo cual coincide con lo reportado por Castellanelli et al. (2008), quienes obtienen un leve incremento en la potencia para mezclas menores a B50 en el motor trabajando a velocidades entre 1800 y 2600 RPM. Estos resultados concuerdan con algunas de las mezclas evaluadas en esta investigación que presentan un incremento en la potencia del $4.2 \%$ y $1.3 \%$ para PB 5/HB2.5/AFUB2.5/D 90 (B 10) y PB5/HB5/AFUB10/D80 (B20) respectivamente. Además, Sahoo et al. (2009) también reportan un incremento en la potencia del $1.5 \%$ y $3.5 \%$ para B20 y B50 respectivamente, con biodiesel de jatropha en un motor de 60HP a 2100 RPM, condiciones muy similares a las de la presente investigación. Sin embargo, se encontraron mezclas que presentaron una importante reducción de la potencia como las mezclas AFUB $10 /$ D 90 con $20.21 \mathrm{~kW} / \mathrm{h}$ y PB6.66/HB6.66/AFUB6.66/D80 con 21.36
$\mathrm{kW} / \mathrm{h}$, entre otras. En términos generales, se encontró que las mezclas B20 y B10 muestran mayor reducción de la potencia, con valores de $26.56 \mathrm{~kW}$ y $27.88 \mathrm{~kW}$, lo que representa una reducción porcentual en la potencia del $16.8 \%$ y $12.6 \%$, respecto al D100, respectivamente; mientras que las mezclas B30 mostraron la menor disminución promedio de la potencia con 30.04 $\mathrm{kW}$ en comparación con el $31.93 \mathrm{~kW}$ del D100. Las mezclas PB10, PB20 y PB30 siempre mostraron valores inferiores de potencia respecto al D100 (31.93 kW), excepto las mezclas P B 5/H B 5/A F U B 1 0/D 80 y $\mathrm{PB} 5 / \mathrm{HB} 2.5 / \mathrm{AFUB} 2.5 / \mathrm{D} 90$ que reportan valores superiores de 32.35 y $33.26 \mathrm{~kW}$ respectivamente.

Consumo específico de combustible (CEC). En relación al consumo específico de combustible se observa que las mezclas B10 (Tabla 4) y B20 (Tabla 5) presentan un consumo promedio mayor, con 241.97 y $256.55 \mathrm{~g} / \mathrm{kWh}$, respectivamente, en comparación con $228.66 \mathrm{~g} / \mathrm{kWh}$ del petrodiesel y $234.5 \mathrm{~g} / \mathrm{kWh}$ de las mezclas de $\mathrm{PB} / \mathrm{D}$ (promedio entre PB10/D90, PB20/D80 y PB30/D70). Para las mezclas de B30 (Tabla 6) se obtuvo el menor aumento en el consumo promedio con $4.16 \%$ 
respecto al presentado por el petrodiesel, mientras que para las mezclas B10 y B20 fue de 5.76 y $10.29 \%$ respectivamente. Por lo tanto, los incrementos en el consumo específico de combustible fueron mayores en las mezclas en relación al petrodiesel, lo cual se puede atribuir, según Moreno et al (1999), a diferencias en el poder calorífico y a las variaciones de viscosidad y densidad que afectan el quemado del combustible. De igual manera Allen \& Watts (2000) encontraron que estas diferencias son generadas por el contenido variable de glicéridos que afecta el patrón de atomización en los inyectores de combustible de los cilindros del motor. Es importante destacar que la mezcla que presentó el mayor consumo de combustible fue HB20/D80 con $279.27 \mathrm{~g} / \mathrm{kWh}$ y la de menor consumo fue PB20/D80 con 213.8 g/kWh, seguida de HB5/PB2.5/AFUB2.5/D90 con $218.97 \mathrm{~g} / \mathrm{kWh}$, comparadas con los $228.66 \mathrm{~g} / \mathrm{kWh}$ del petrodiesel. Estas diferencias se deben básicamente a que el biodiesel de higuerilla presenta valores de viscosidad y densidad superiores a los reportados para los otros biodiesel, y un poder calorífico mucho menor. Aunque las mezclas PB10/D90 y PB30/D70 muestran un consumo específico de combustible mayor al D100, la mezcla PB20/D80 es la de menor consumo, pero este valor no presenta diferencias significativas respecto al consumo de la mezcla HB5/PB2.5/AFUB2.5/D90, por lo tanto esta última mezcla representa mayor importancia en la investigación, ya que dicha mezcla cumple con el propósito de encontrar una muestra que supere o iguale el comportamiento tanto del D100 como de las mezclas $\mathrm{PB} / \mathrm{D}$.

Al comparar simultáneamente los valores promedios de potencia y consumo específico de combustible de las diferentes mezclas de biodiesel (B10, B20 y B30) con los resultados del petrodiesel puro, como se muestra en la Figura 2, se encontró que las mezclas B30 en promedio, presentan gran similitud con el comportamiento del D100, aunque sin superarlo. Por el contrario, las mezclas B20 en promedio, reportaron la menor potencia en el motor con el mayor consumo de combustible.

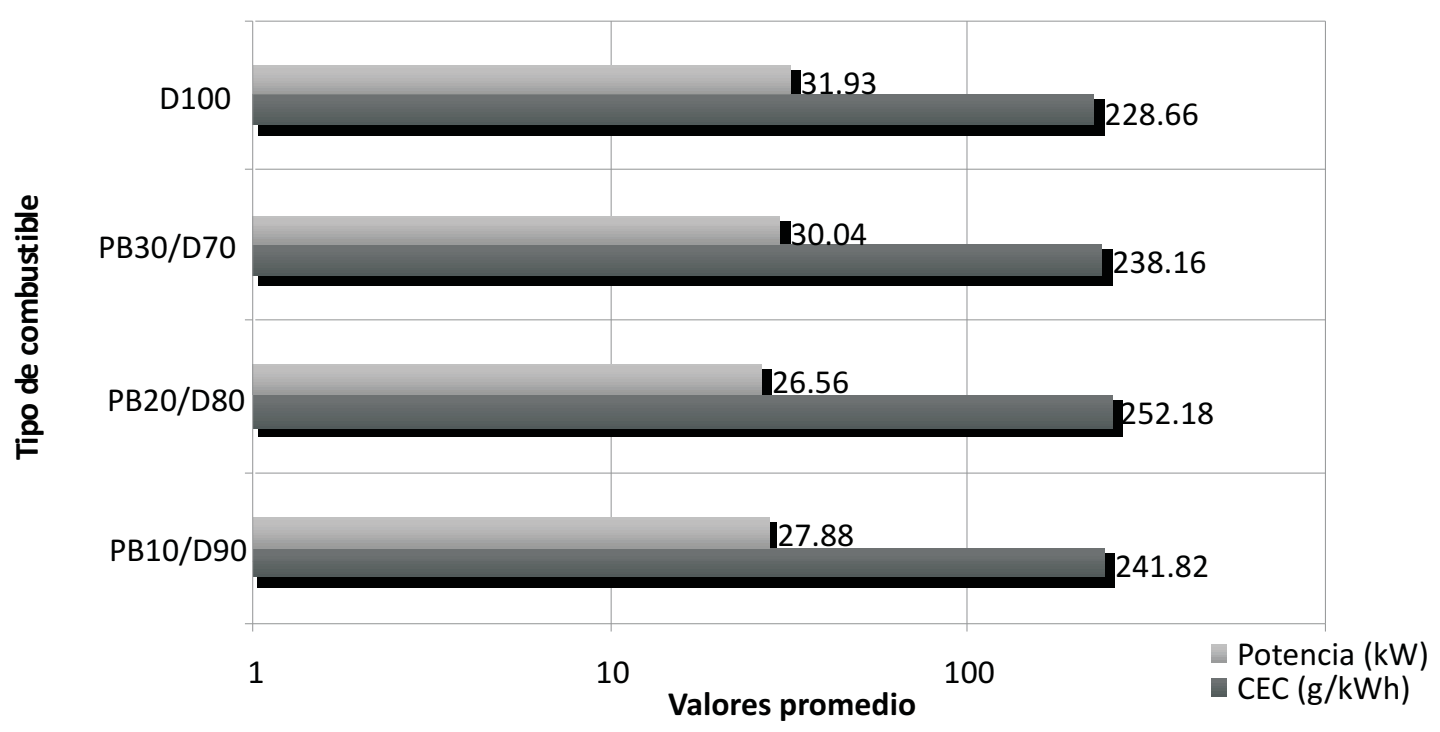

Figura 2. Comparación de valores promedios de potencia y CEC presentados por las mezclas de biodiesel y el Petrodiesel 


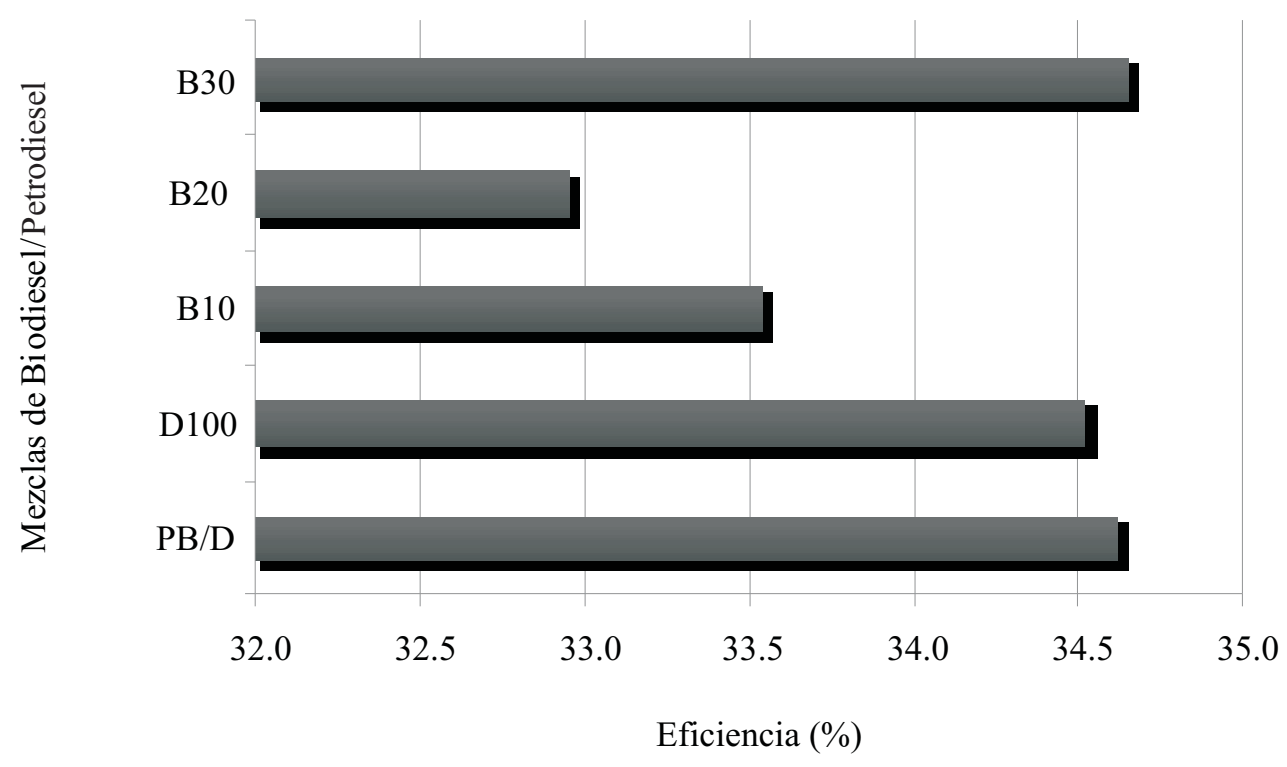

Figura 3. Efecto del contenido de Biodiesel sobre la eficiencia del motor

Eficiencia (E). En la Figura 3 se muestra el efecto del contenido de biodiesel sobre la eficiencia del motor. En esta figura se que las mezclas B10 y B20 muestran valores menores en promedio, de 33.54 y $32.95 \%$ respectivamente, en comparación con el 34.52 y $34.62 \%$ del D100 y las mezclas PB/D, respectivamente. Por el contrario las mezclas B30 (Tabla 6) mostraron un leve incremento promedio en la eficiencia de $0.37 \%$ con respecto al D100 y del $0.09 \%$ con respecto a las mezclas $\mathrm{PB} / \mathrm{D}$ (Figura 3). En relacion a este parámetro la mezcla menos eficiente fue PB5/HB5/D90 con $28.7 \%$, y la de mayor eficiencia fue la mezcla PB20/D80 $\begin{array}{llllll}\text { c o n } & 37.7 \% & \text { s e g u i d a d e }\end{array}$ PB6.66/HB6.66/AFUB6.66/D80 con 37.07\%. $\mathrm{Cabe}$ resaltar que $1 \mathrm{a}$ mezcla PB5/HB5/AFUB10/D80, además de ser una de las que genera mayor potencia $(32.35 \mathrm{~kW})$ y menor consumo específico de combustible (224.83 $\mathrm{g} / \mathrm{kWh}$ ), también está entre las mezclas más eficientes con un valor de $36.08 \%$.

Análisis de emisiones gaseosas. Los resultados del análisis Orsat de los biocombustibles B10, B20 y B30 se presentan en las Tablas 7, 8 y 9 respectivamente. Los resultados muestran una disminución del porcentaje de $\mathrm{CO}_{2}$ en promedio para las mezclas B10 (Tabla 7) y B20 (Tabla 8), cuyos valores son de $8.63 \%$ y $7.54 \%$ respectivamente, comparados con el $8.95 \%$ del petrodiesel y el $7.85 \%$ de las mezclas PB/D. Sin embargo, las mezclas de B30 (Tabla 8) presentaron un incremento relativamente alto respecto al valor promedio del petrodiesel y las mezclas $\mathrm{PB} / \mathrm{D}$ con $9.68 \%$, lo cual indica una mejor combustión. La mezcla PB10/HB10/AFUB10/D70 fue la que presentó la mayor producción de $\mathrm{CO}_{2}$ con un valor de $11.50 \%$, lo que representa un aumento del $28.5 \%$ con respecto al $\mathrm{CO}_{2}$ producido por el petrodiesel y de $46.5 \%$ respecto al promedio de las mezclas PB30/D70 (B30), mientras que la mezcla que produjo el menor $\mathrm{CO}_{2}$ con un valor de $3.5 \%$ fue AFUB30/D70. En cuanto a la producción de CO se encontró un incremento para las mezclas de B20, cuyo valor promedio fue de $8.79 \%$ de $\mathrm{CO}$ comparado con el $6.12 \%$ del petrodiesel y una reducción del $12.13 \%$ respecto a la mezcla de $\mathrm{PB} / \mathrm{D}$; mientras que para las mezclas de $\mathrm{B} 10$ y B30 se encontraron valores promedios de $\mathrm{CO}$ muy superiores al del petrodiesel y las mezclas $\mathrm{PB} / \mathrm{D}$, con 14.92 y $16.12 \%$, respectivamente. Según Sahoo et al. (2009) encontraron un aumento cercano al 35\% de CO con mezclas de B20, B50 y $\mathrm{B} 100$, esto se debe a factores que causan el deterioro de la combustión como lo son el alto 
Ingeniería y Competitividad, Volumen 11, No. 2, p. 63 - 78 (2009)

Tabla 7. Composición de gases de combustión para las mezclas B10

\begin{tabular}{lcccc}
\hline Mezcla & $\mathbf{C O}_{2}(\mathbf{\%})$ & $\mathbf{O}_{2}(\mathbf{\%})$ & $\mathbf{C O}(\%)$ & $\begin{array}{c}\text { Otros } \\
\text { gases (\%) }\end{array}$ \\
\hline D100 & 8.50 & 5.77 & 6.12 & 79.17 \\
PB10-D90 & 8.00 & 11.68 & 12.62 & 67.70 \\
HB10-D90 & 7.65 & 12.62 & 14.25 & 65.48 \\
AFUB10-D90 & 8.00 & 13.26 & 15.35 & 63.39 \\
PB5-HB5-D90 & 10.50 & 15.08 & 17.11 & 57.31 \\
HB5-AFUB5-D90 & 7.90 & 12.32 & 14.24 & 65.53 \\
PB5-AFUB5-D90 & 9.50 & 14.09 & 16.72 & 59.69 \\
PB5-HB2.5-AFUB2.5-D90 & 7.50 & 12.27 & 14.48 & 65.75 \\
PB2.5-HB5-AFUB2.5-D90 & 9.15 & 13.32 & 15.49 & 62.04 \\
PB2.5-HB2.5-AFUB5-D90 & 9.65 & 14.67 & 16.80 & 58.89 \\
PB3.33-HB3.33-AFUB3.33-D90 & 8.45 & 11.14 & 12.17 & 68.24 \\
\hline
\end{tabular}

Tabla 8. Composición de gases de combustión para las mezclas B20

\begin{tabular}{lcccc}
\hline Mezcla & $\mathbf{C O}_{\mathbf{2}}(\mathbf{\%})$ & $\mathbf{O}_{2}(\mathbf{\%})$ & $\mathbf{C O}(\%)$ & $\begin{array}{c}\text { Otros } \\
\text { gases (\%) }\end{array}$ \\
\hline D100 & 8.95 & 5.77 & 6.12 & 79.17 \\
PB20-D80 & 5.35 & 5.42 & 5.89 & 84.69 \\
HB20-D80 & 7.10 & 7.54 & 7.99 & 80.56 \\
AFUB20-D80 & 4.40 & 6.20 & 5.29 & 77.27 \\
PB10-HB10-D80 & 7.10 & 7.83 & 8.15 & 78.52 \\
PB10-AFUB10-D80 & 7.40 & 8.77 & 8.62 & 76.65 \\
HB10-AFUB10-D80 & 10.40 & 10.40 & 12.38 & 70.97 \\
PB10-HB5-AFUB5-D80 & 10.25 & 10.61 & 12.48 & 68.81 \\
PB5-HB10-AFUB5-D80 & 7.60 & 8.32 & 8.90 & 75.87 \\
PB5-HB5-AFUB10-D80 & 7.75 & 7.63 & 8.83 & 78.54 \\
PB6.66-HB6.66-AFUB6.66-D80 & 8.00 & 8.76 & 9.36 & 75.53 \\
\hline
\end{tabular}


Ingeniería y Competitividad, Volumen 11, No. 2, p. 63 - 78 (2009)

Tabla 9. Composición de gases de combustión para las mezclas B30

\begin{tabular}{lcccc}
\hline Mezcla & $\mathbf{C O}_{2}(\mathbf{\%})$ & $\mathbf{O}_{2}(\mathbf{\%})$ & $\mathbf{C O}(\%)$ & $\begin{array}{c}\text { Otros } \\
\text { gases (\%) }\end{array}$ \\
\hline D100 & 8.95 & 5.77 & 6.12 & 79.17 \\
PB30-D70 & 10.20 & 14.48 & 17.90 & 57.42 \\
HB30-D70 & 10.00 & 14.44 & 16.56 & 59.00 \\
AFUB30-D70 & 3.50 & 4.56 & 4.34 & 87.60 \\
PB15-HB15-D70 & 9.75 & 15.29 & 15.37 & 59.59 \\
PB15-AFUB15-D70 & 9.70 & 14.12 & 19.34 & 56.84 \\
HB15-AFUB15-D70 & 9.60 & 13.83 & 15.66 & 60.91 \\
PB15-HB7.5-AFUB7.5-D70 & 11.25 & 16.90 & 20.88 & 50.97 \\
PB7.5-HB15-AFUB7.5-D70 & 10.50 & 14.69 & 16.70 & 58.11 \\
PB7.5-HB7.5-AFUB15-D70 & 10.75 & 13.39 & 14.94 & 60.92 \\
PB10-HB10-AFUB10-D70 & 11.50 & 16.16 & 19.54 & 52.80 \\
\hline
\end{tabular}

calor latente de evaporación y el aumento de la viscosidad al ampliar la concentración de biodiesel en las mezclas, lo que conlleva a la obtención de un combustible menos homogéneo. Finalmente, es importante definir que la mezcla PB3.33/HB3.33/AFUB3.33/D90 presenta una mejor combustión comparada con PB10/D90, ya que mostró un importante aumento en la producción de $\mathrm{CO}_{2} \mathrm{y}$ una leve disminución en el CO. En cuanto a las mezclas B20 en general, ninguna mezcla presentó una mejor combustión respecto a PB20/D80, debido a que se incrementó en gran proporción la producción tanto de $\mathrm{CO}_{2}$ como de CO, pero la mezcla AFUB20/D80 expuso un comportamiento muy similar al de PB20/D80, aunque sin superarla. De igual manera para las $\mathrm{m} \mathrm{e} \mathrm{z} \mathrm{c} \mathrm{la} \mathrm{s} \mathrm{B} 30,1 \mathrm{a} m \mathrm{u}$ e s t r a PB7.5/HB7.5/AFUB15/D70 evidenció una mejora en la combustión comparado con la mezcla PB30/D70, debido a que presentó un leve aumento en la producción de $\mathrm{CO}_{2}$ y una significativa disminución del CO.

\section{Conclusiones}

Los resultados de la caracterización físico-química de las mezclas mostraron que el BP100 cumplió con la totalidad de los parámetros evaluados y contemplados en la NTC 5444 ya que esta norma fue establecida en función de este tipo de biodiesel; mientras que el HB100 y AFUB100 no cumplieron con algunos de los parámetros como viscosidad e índice de acidez, pero cuando se analizan las mezclas conformadas en diferentes proporciones por estos tipos de biodiesel muestran una importante similitud con la mezcla comercial de PB20/D80, haciendo posible la viabilidad de producirlas y comercializarlas, así el biodiesel puro que las conforman no cumplan la norma en su totalidad.

Al comparar los dos aspectos evaluados (composición de gases y consumo de combustible) en el motor estacionario KIPOR, se destacó la mezcla PB10/HB10/D80 debido a que presentó el menor consumo de combustible y una composición de gases de ignición similar a la del D100 y menor a la de PB20/D80.

En relación al motor MASSEY-FERGUSON, la mezcla que presentó el mejor comportamiento en cuanto a potencia y consumo de combustible fue la 
conformada por PB5/HB5/AFUB10/D80 con $32.35 \mathrm{~kW}$ de potencia y $224.83 \mathrm{~g} / \mathrm{kWh}$ de consumo, estos valores superaron el comportamiento del D100 y el de las mezclas $\mathrm{PB} / \mathrm{D}$. Además, esta mezcla mostró ser una de las más eficientes en la combustión con un valor del $36.08 \%$.

Respecto a la composición de los gases de c o m b u s t i ó,$\quad 1$ a m e z c 1 a PB3.33/HB3.33/AFUB3.33/D90 presentó una mejor combustión comparada con PB10/D90, ya que mostró un importante aumento en la producción de $\mathrm{CO}_{2}$ y una leve disminución en el CO. En las mezclas B20 en general, se incrementó en gran proporción la producción tanto de $\mathrm{CO}_{2}$ como de CO, respecto a la mezcla PB20/D80. De igual manera para las mezclas de Biodiesel 30\%, la de PB7.5/HB7.5/AFUB15/D70 evidenció una mejora en la combustión comparándola con la PB30/D70, debido a que presentó un leve aumento en la producción de $\mathrm{CO}_{2}$ y una significativa disminución del CO.

\section{Agradecimientos}

Los autores agradecen a la oficina de Investigación de la Universidad Nacional de Colombia Sede Palmira-DIPAL, por la financiación del proyecto. De igual manera agradecen al ingeniero Cesar Augusto Medina y al señor Carlos Pinchao por su gran apoyo técnico al proyecto.

\section{Referencias bibliográficas}

Agudelo, J.R., Benjumea P., Gómez E., \& Pérez J.F. (2003). Biodiesel: una revisión del desempeño mecánico y ambiental. Revista Ingeniería \& Desarrollo, Universidad del Norte, 13,1-14.

Allen, C., \& Watts, K. (2000). Comparative analysis of the atomization characteristics of fifteen biodiesel fuel types. Transaction of the ASAE 43 (2), 207-211.

Barbosa, R., Da Silva, F., Salvador, N., \& Volpato, C. (2008). Comparative performance of a cycle diesel engine using diesel and biodiesel mixtures. Ciência e Agrotecnología 32 (5), 1588-1593.
Canakci, M., Ozsezen, A. N., Arcaklioglu, E., \& Erdil A. (2009). Prediction of performance and exhaust emissions of a diesel engine fueled with biodiesel produced from waste frying palm oil. Experts y Sytems with Applications 36(5), 92689280.

Castellanelli, M., De Souza, S., Silva, S., \& Kailer, E. (2008). Performance of cicle diesel engine in dynamometer using diesel/biodiesel mixtures. Engenharia Agrícola Jaboticabal 28 (1), 145-153.

Correa, I., González, J., Úngaro, M., Bernardi, J., \& Storino, M. (2008). Desempenho de motor diesel com misturas de biodiesel de óleo de girasol. Ciência e Agrotecnología 32 (3), 923-928.

Herrero, R., \& Maiza I. (2008). Influencia de los biocombustibles sobre los materiales plásticos empleados en automoción. Centro Multidisciplinar de Innovación y Tecnología de Navarra. España, 9-13.

Hilbert, J., Tesouro, M., Aucaná, M., \& Pincu, M. (2006). Rendimiento comparativo de biodiesel y gasoil en tractores agrícolas. Instituto de Ingeniería Rural INTA. Castelar, Argentina.

Homer, I. Motores y Biocombustibles. (2006). In: Acevedo, E., Agroenergía. Un desafió para Chile. Serie agronómica (11), Universidad de Chile, 149-164.

Issariyakul T., Kulkarni M. G., Meher L. C., Dalai A. K. \& Bakhshi N. N. (2008). Biodiesel production from mixtures of canola oil and used cooking oil. Chemical Engineering Journal 140(1-3), 77-85.

Kalscheuer, R., Torsen, S., \& Steinbuchel, A. (2006). Microdiesel: Escherichia coli engineered Microbiology 152, 2529-2536.

Meher, L., Vidya, S., \& Naik, S. (2006). Technical aspects of biodiesel production by transesterification-a review. Renowable \& Sustainable Energy Reviews 10 (3), 248-268.

Moreno, F., Muñoz, M., \& Morea-Roy, J. (1999). Sunflower methyl ester as a fuel for automobile diesel engines. Transaction of the ASAE 42 (5), 1181-1185. 
Plentz, S.M., Meneghetti, M.R., Serra, T.M., Barbosa, D.C. \& Wolf, C.R. (2007). Biodiesel Production from Vegetable Oil Mixtures: Cottonseed, Soybean, and Castor Oils. Energy \& Fuels 21(6), 3746-3747.

Sahoo, P., Das, L., Babu, M., Arora, P., Singh, V., Kumar, N., \& Varyani, T. (2009). Comparative evaluation of performance and emission characteristics of jatropha, karanja and polanga based biodiesel as fuel in a tractor engine. Fuel 88 (4), 1698-1707.

Sarin, R., Sharma, S., Sinharay, M., \& Malhotra, R.(2006). Jatropha-Palm biodiesel blends: An optimum mix for Asia. Fuel 86(10-11), 13651371.

Tinaut, F., Castaño, V., \& Lapuerta, M. (1999). Bioenergía. España: Gamesal editores.

Zhang, Y., Dubé, M., McLean, D., \& Kates, M. (2003). Biodiesel production from waste cooking oil: 1. Process design and technological assessment. Bioresource Technology 89 (1), 1-16. 